\title{
Explicit $O\left(h^{2}\right)$ Bounds on the Eigenvalues of the Half-L*
}

\author{
By Blair K. Swartz
}

0. Summary and Survey. This paper is concerned with obtaining strict upper and lower bounds on the eigenvalues of a particular nontrivial convex membrane, the half- $L$, which is fixed or free at the boundary. The upper bound is obtained from a matrix eigenvalue calculation; the matrix problem may be regarded as a difference scheme although it is derived using piecewise linear functions in a Rayleigh quotient. The lower bound is than calculated from the upper bound using an elementary formula. The validity of this formula is proved by extensions of Weinberger's techniques [3]. Difficulties encountered in determining similar results for the nonconvex $L$-shaped membrane are indicated. A numerical example illustrates the results. An appendix contains some pointwise bounds on normalized eigenfunctions.

An annotated survey of the literature concerned with estimating the eigenvalues of the Laplacian is contained in [4]; many other references are found in [10]. The use of piecewise linear functions in variational principles is discussed in [1], [2], [5]; piecewise bilinear in [6], [7], [9, pp. 331-334]. A general discussion of Rayleigh-Ritz eigenvalues is found in [8].

Much of the literature concerned with strict bounds on the eigenvalues seems to use the eigenvalues of the discrete Laplacian or a related matrix rather than the eigenvalues associated with the Rayleigh-Ritz method. The lower bounds of [19], [3], [13], and the simultaneous two-sided bounds in [10] are $O(h)$ bounds as a result of embedding a general region in a union of squares [9, p. 339], [4, p. 30]. It is not clear how the lower bound in [14] behaves as $h \rightarrow 0$. (See also [4, pp. 30-31].) Other results have been asymptotic: the discrete eigenvalue is the continuous one (in certain cases) except for a term $\gamma h^{2}+o\left(h^{2}\right), \gamma$ unknown [4], [9]; a result quite useful in extrapolation to zero.

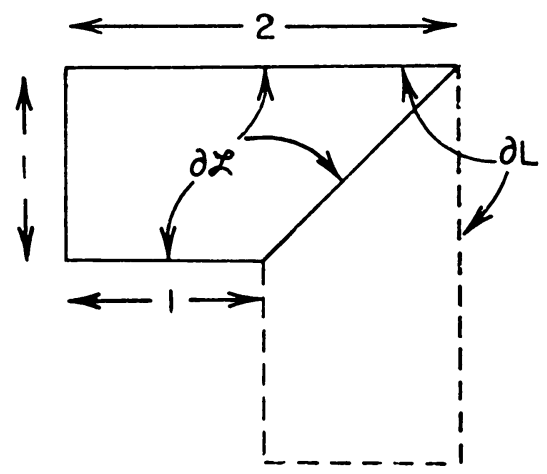

Figure 1

Received November 15, 1965. Revised May 8, 1967.

* Work performed under AEC contract No. W-7405-ENG-36. 
1. General Approach, Results, and Notation. We shall be concerned throughout with two regions in the plane (Fig. 1).

The primary interest will be in the lowest nontrivial eigenvalue of two problems:

I. $\Delta u=-\lambda u$ in $\mathscr{L}, u=0$ on $\partial \mathscr{L}$ (Sections 2 and 3 , two different meshes); and

II. $\Delta u=-\lambda u$ in $\mathscr{L}, \partial u / \partial \nu=0$ on $\partial \mathscr{L}$ (Section 4 ).

However, the bounds on $\lambda$ are shown to hold for the higher eigenvalues as well (Section 5).

The general approach is a variational one. In each case

$$
\lambda=\min _{w} \frac{\iint|\nabla w|^{2} d A}{\iint w^{2} d A}
$$

where $W$ is a class of continuous, piecewise differentiable functions satisfying the boundary conditions. The approximating procedure consists of placing a mesh on the region in a particular manner, triangulating it, and considering a class $V$ of continuous functions, piecewise linear on the triangles, satisfying the boundary condition. If $\mathbf{x}_{i}$ are the interior mesh points, each $v \in V$ may be represented by a vector $\mathbf{v}=\left\{v\left(\mathbf{x}_{i}\right)\right\}$. We then have, with $\iint|\nabla v|^{2} d A \equiv N(\mathbf{v}) \equiv(\mathbf{v}, A \mathbf{v}), \iint v^{2} d A \equiv D(\mathbf{v}) \equiv$ $(\mathbf{v}, B \mathbf{v})$;

$$
\lambda \leqq \lambda_{h}=\min _{v \in v} \frac{\iint|\nabla v|^{2} d A}{\iint v^{2} d A}=\min _{\mathbf{v}} \frac{N(\mathbf{v})}{D(\mathbf{v})}=\min _{\mathbf{v}} \frac{(\mathbf{v}, A \mathbf{v})}{(\mathbf{v}, B \mathbf{v})}
$$

( $N$ and $D$ vary from section to section as the meshes and/or problem vary.) It is easily shown that $A$ and $B$ are symmetric positive-definite sparse matrices. $(A \mathbf{v}$, for example, will in each case be essentially $-h^{2} \Delta_{h} \mathbf{v}$.) The numerical calculation of $\lambda_{h}$ then amounts to solving $A \mathbf{v}=\lambda_{h} B \mathbf{v}$, which can be done by successive over-relaxation, as indicated in Section 7.

To obtain the lower bound on $\lambda$ in terms of $\lambda_{h}$ one shows three things. With $u$, the eigenfunction, normalized so that $\iint u^{2} d A=1$, define

$$
\bar{u}(\mathbf{x})=\iint_{S(\mathbf{x})} u d A / h^{2}
$$

where $S(\mathbf{x})$ is a square of side $h$ centered on $\mathbf{x}$, and set $\mathbf{u}=\left\{\bar{u}\left(\mathbf{x}_{i}\right)\right\}$. For Problem I it is then shown that

$$
\begin{aligned}
D(\mathbf{u}) & \geqq h^{2}(\mathbf{u}, \mathbf{u})-h^{2} N(\mathbf{u}) / 4, \\
h^{2}(\mathbf{u}, \mathbf{u}) & \geqq 1-h^{2} \lambda / \pi^{2}, \quad \text { and } \\
N(\mathbf{u}) & \leqq \lambda .
\end{aligned}
$$

As an immediate consequence we have, with $c=1 / 4+1 / \pi^{2}$ and $c h^{2} \lambda<1$,

$$
\lambda \leqq \lambda_{h} \leqq \lambda /\left(1-c h^{2} \lambda\right),
$$

indicating that $\lambda_{h}-\lambda \leqq O\left(h^{2}\right)$. Solving (1.4) for $\lambda$ yields the explicit double bounds

$$
\lambda_{h} /\left(1+\operatorname{ch}^{2} \lambda_{h}\right) \leqq \lambda \leqq \lambda_{h}
$$

Finally, we show the same result holds for the higher eigenvalues, $\lambda^{(k)}$ :

$$
\lambda_{h}{ }^{(k)} /\left(1+\operatorname{ch}^{2} \lambda_{h}{ }^{(k)}\right) \leqq \lambda^{(k)} \leqq \lambda_{h}{ }^{(k)} \text { if } \quad \operatorname{ch}^{2} \lambda_{h}{ }^{(k)}<1 .
$$

For problem II it is necessary to subtract an additional term, $h^{2} u_{0}^{2} / 4$, from (1.1), 
where $u_{0}$ is a bound on the normalized eigenfunction in certain subsets of $\mathscr{L}$. (1.4) then becomes

$$
\lambda \leqq \lambda_{h} \leqq \lambda /\left(1-\operatorname{ch}^{2} \lambda-h^{2} f(\lambda, h) / 4\right) \equiv g_{h}(\lambda) .
$$

(See Appendix IV, Eq. (IV.2).) Since $f(\lambda, h)$ is increasing in $\lambda$ for each $h, g$ has the same property. One then concludes, with $\lambda$ the first nontrivial eigenvalue of problem II,

$$
g_{h}^{-1}\left(\lambda_{h}\right) \leqq \lambda \leqq \lambda_{h} ;
$$

$g_{h}$ is easily inverted numerically. The higher eigenvalues may be bounded below by inverting a corresponding function (5.1), given upper bounds $\lambda_{h}{ }^{(1)}, \cdots, \lambda_{h}{ }^{(k)}$.

We now turn to particulars.

2. $\Delta u=-\lambda u, u=0$ on $\partial \mathcal{L}$. For reasons to become apparent we place a mesh on the full $L$ and triangulate it as in Fig. 2.

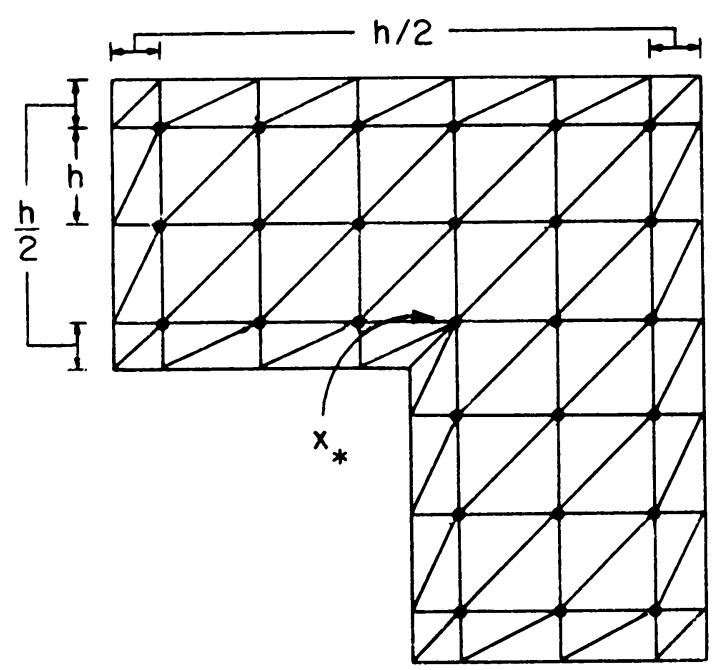

Figure 2

All $v$ vanish on the boundary and hence are determined by their values on the $\mathbf{x}_{i}$ (the dots).

$N(\mathbf{v})$ and $D(\mathbf{v})$ are indicated in Appendix I, where it is also shown that

$$
D(\mathbf{v}) \geqq h^{2}(\mathbf{v}, \mathbf{v})-h^{2} N(\mathbf{v}) / 4 \text {. }
$$

We now substitute the averaged eigenfunction $\mathbf{u}=\left\{\iint_{S_{i}} u d A / h^{2}\right\} \equiv\left\{u_{i}\right\}$, where $S_{i}=S\left(\mathbf{x}_{i}\right)$. For the time being we assume $u(\lambda)$ is the first normalized eigenfunction (eigenvalue) of the full L. To show

$$
h^{2}(\mathbf{u}, \mathbf{u}) \geqq 1-h^{2} \lambda / \pi^{2}
$$

we sum the inequality

$$
h^{2} u_{i}{ }^{2} \equiv h^{2}\left(\iint_{S_{i}} \frac{u d A}{h^{2}}\right)^{2} \geqq \iint_{S_{i}} u^{2} d A-\frac{h^{2}}{\pi^{2}} \iint_{S_{i}}|\nabla u|^{2} d A
$$


and note the union of the $S_{i}$ is $L$.

This last inequality is a result of Weinberger [3, p. 342] and follows from the fact that $u-u_{i}$ is orthogonal over $S_{i}$ to all constant functions. Hence, it may be substituted in the Rayleigh quotient for the first nontrivial Neumann eigenfunction for $S_{i}$, yielding

$$
\frac{\pi^{2}}{h^{2}} \leqq \frac{\iint s_{i}|\nabla u|^{2} d A}{\left(\iint_{S_{i}} u^{2} d A-h^{2} u_{i}{ }^{2}\right)},
$$

which is equivalent to $(2.21)$.

It remains to show $N(\mathbf{u}) \leqq \lambda$.

$N(\mathbf{u})$ may be regarded as composed primarily of sums of squares of horizontal and vertical differences of the $\bar{u}\left(\mathbf{x}_{i}\right)$. To estimate them, let $H$ be any horizontal line through the interior mesh points, extending to the boundary (Fig. 3).

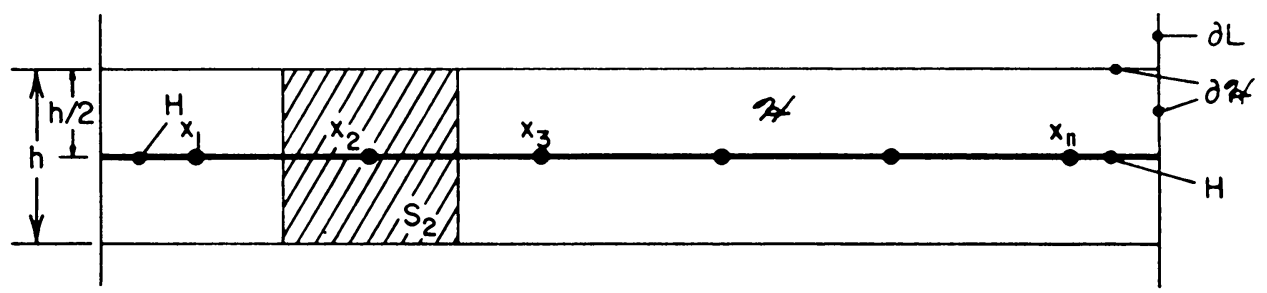

Figure 3

Regard the $\mathbf{x}_{i}$ in $H$ as ordered $1 \leqq i \leqq n$ from left to right. Then set

$$
\sigma_{H}=2 \bar{u}^{2}\left(\mathbf{x}_{1}\right)+\sum_{i=1}^{n-1}\left(\bar{u}\left(\mathbf{x}_{i+1}\right)-\bar{u}\left(\mathbf{x}_{i}\right)\right)^{2}+2 \bar{u}^{2}\left(\mathbf{x}_{n}\right) .
$$

Let $V$ be a similar vertical line, and define $\sigma_{V}$ similarly. Appendix I shows that $N(\mathbf{u})$ $\leqq \sum_{\mathrm{all} H} \sigma_{H}+\sum_{\mathrm{all} V} \sigma_{V}+\bar{u}^{2}\left(\mathbf{x}_{*}\right)$ where $\mathbf{x}_{*}$ is the mesh point $N E$ of the re-entrant corner (see Fig. 2).

Furthermore, let $\mathfrak{F C}$ be the strip of height $h$ centered on $H$. Then $\bigcup_{a 11} \mathfrak{F} \mathfrak{F}=L$ and, similarly, $\cup_{\mathrm{all}} v=L$.

We now show $\sigma_{H} \leqq \iint \mathfrak{C} u_{x}^{2} d A$. From this it follows that (since the $\mathfrak{F C}$ are disjoint) $\sum_{\mathrm{all} H} \sigma_{H} \leqq \iint_{L} u_{x}^{2} d A$, and similarly $\sum_{\mathrm{all} V} \sigma_{V} \leqq \iint_{L} u_{y}^{2} d A$. Hence

$$
N(\mathbf{u}) \leqq \lambda+\bar{u}^{2}\left(\mathbf{x}_{*}\right) .
$$

Proof that $\sigma_{H} \leqq \iint \mathfrak{F C} u_{x}^{2} d A$.

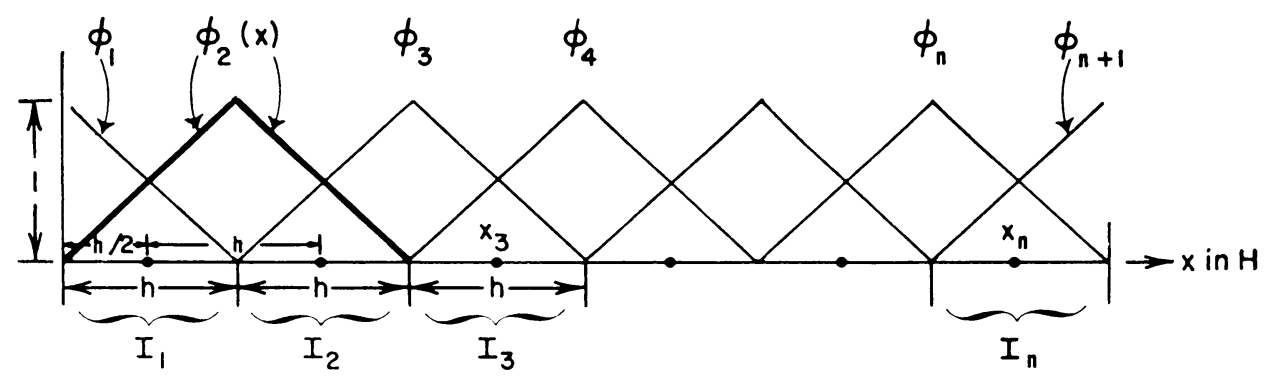

Figure 4 
With Weinberger [3, p. 343], define $\phi_{i}(x), i=1,2, \cdots, n+1$ as in Fig. 4. Observe that $\left|\phi_{x}\right|=1 / h$. Hence, integrating by parts,

$$
\begin{aligned}
\int_{I_{i+1}} \frac{u}{h} d x-\int_{I_{i}} \frac{u}{h} d x=-\int_{I_{i+1} \cup I_{i}} u \frac{d \phi_{i+1}}{d x}=\int_{I_{i+1} \cup I_{i}} u_{x} \phi_{i+1} d x ; \\
i=1,2, \cdots, n .
\end{aligned}
$$
have

Dividing by $h$, integrating on $y$ with $y$ going from 0 to $h$ on $\mathfrak{F}$, and squaring, we

$$
\begin{gathered}
\left(\int_{0}^{h} \int_{I_{i+1}} \frac{u}{h^{2}} d x d y-\int_{0}^{h} \int_{I_{i}} \frac{u}{h^{2}} d x d y\right)^{2}=\frac{1}{h^{2}}\left(\int_{0}^{h} \int_{I_{i+1} \mathrm{UI} I_{i}} \phi_{i+1} u_{x} d x d y\right)^{2} \\
\leqq \frac{1}{h^{2}} \int_{0}^{h} \int_{I_{i+1} \mathrm{U} I_{i}} \phi_{i+1} d A \iint \phi_{i+1} u_{x}^{2} d A
\end{gathered}
$$

the last by Schwarz inequality. Since

$$
\int_{0}^{h} \int_{I_{i+1} \mathrm{UI} I_{i}} \phi_{i+1} d A=h^{2}
$$

we have

$$
\left(\bar{u}\left(\mathbf{x}_{i+1}\right)-\bar{u}\left(\mathbf{x}_{i}\right)\right)^{2} \leqq \int_{0}^{h} \int_{I_{i+1} \cup I_{i}} \phi_{i+1} u_{x}^{2} d A
$$

As for the ends (take the left one),

$$
\int_{I_{1}} \frac{u}{h} d x=-\int_{I_{1}} u \frac{d \phi_{1}}{d x}=\int_{I_{1}} u_{x} \phi_{1} d x
$$

where we have used the fact that $u=0$ at left boundary of $\mathfrak{K}$; i.e., the left end of $I_{1}$. Hence

$$
2 \bar{u}^{2}\left(\mathbf{x}_{1}\right)=\frac{2}{h^{2}}\left(\int_{0}^{h} \int_{I_{1}} u_{x} \phi_{1} d A\right)^{2} \leqq \frac{2}{h^{2}} \iint \phi_{1} d A \iint \phi_{1} u_{x}^{2} d A=\int_{0}^{h} \int_{I_{1}} \phi_{1} u_{x}^{2} d A .
$$

Summing, we have, since $\sum \phi_{i} \equiv 1, \sigma_{H} \leqq \iint_{\mathfrak{C}} u_{x}^{2} d A$.

We now conclude from (2.1), (2.2), (2.3), with $\mathbf{x}_{*}$ as in Fig. 2,

$$
\lambda_{h} \leqq \frac{\lambda+\bar{u}^{2}\left(\mathbf{x}_{*}\right)}{1-c \lambda h^{2}-\bar{u}^{2}\left(\mathbf{x}_{*}\right) h^{2} / 4} .
$$

Since $\bar{u}^{2}\left(\mathbf{x}_{*}\right)$ is $O\left(h^{4 / 3}\right)$ for the first eigenfunction of the full $L$ (with unknown constant), we cannot obtain an explicit lower bound for this region. However, this does give an $O\left(h^{4 / 3}\right)$ upper bound on $\lambda_{h}-\lambda$, in analogy to that obtained in [11, Theorem 1, p. 1038], [4, p. 82] for the eigenvalue of the usual discrete Laplacian.

However, if we consider the first eigenfunction of the half- $L, \mathcal{L}$; and redefine $u$ to be this eigenfunction reflected oddly in the diagonal of $L$, we have $\bar{u}\left(\mathbf{x}_{*}\right)=0$. We assume $u$ is normalized over the full $L$.

Redefining $\lambda_{h}=\min _{V_{2}} N(\mathbf{v}) / D(\mathbf{v})$, where the $\mathbf{v} \in V_{2}$ are antisymmetric in the diagonal and vanish on it, we have, by (2.1), (2.2), and (2.3),

$$
\lambda \leqq \lambda_{h} \leqq \lambda /\left(1-c h^{2} \lambda\right), \quad c=1 / 4+1 / \pi^{2} .
$$


Thus, for $h$ sufficiently small, $1-c h^{2} \lambda_{h}>0$, and we may conclude

$$
\lambda_{h} /\left(1+c \lambda_{h} h^{2}\right) \leqq \lambda \leqq \lambda_{h}, \quad \lambda=1 \text { st eigenvalue of } \mathscr{L} .
$$

It is a straightforward extension of these arguments to conclude that the first eigenvalue of a horizontally stretched $\mathcal{L}$, approximated by the Rayleigh-Ritz technique using similarly stretched triangles of dimension $h \times k$, satisfies

$$
\lambda_{h} /\left\{1+c[\max (h, k)]^{2} \lambda_{h}\right\} \leqq \lambda \leqq \lambda_{h} .
$$

3. $\Delta u=-\lambda u, u=0$ on $\partial \mathscr{L}$; Standard Mesh. We place the standard mesh on $\mathfrak{L}$ and triangulate as in Fig. 5:

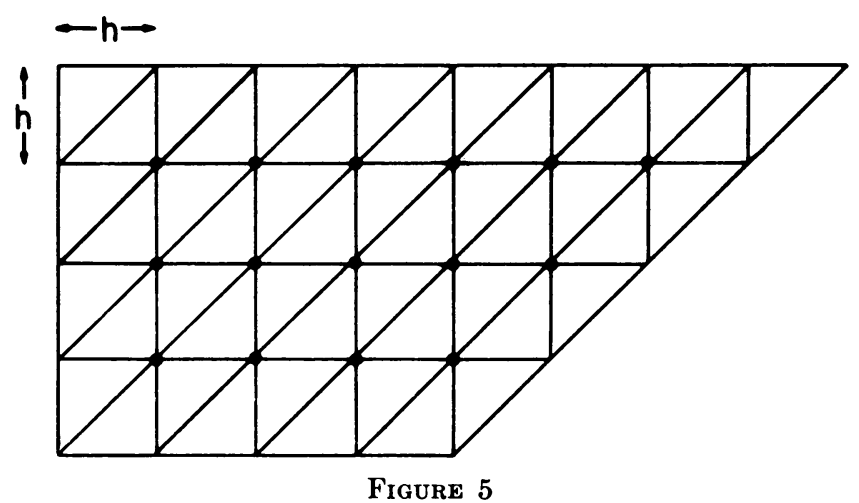

The quadratic forms $N(\mathbf{v})$ and $D(\mathbf{v})$ have changed (Appendix II), and the relations (1.1)-(1.3) must be re-proved. Appendix II shows again, however, that

$$
D(\mathbf{v}) \geqq h^{2}(\mathbf{v}, \mathbf{v})-h^{2} N(\mathbf{v}) / 4 \text {. }
$$

We have difficulty in proving (1.2) because $R=\cup_{\mathbf{x}_{i}} S\left(\mathbf{x}_{i}\right) \neq \mathcal{L} . R$ is shaded in Fig. 6.

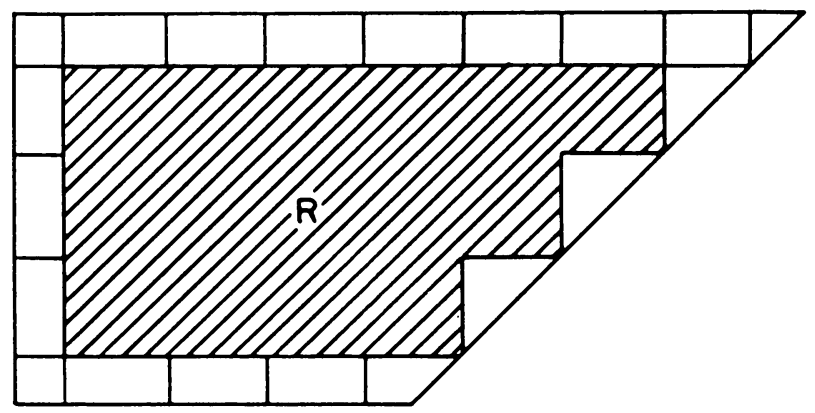

Figure 6

Let $\bar{R}=\mathcal{L}-R$. By (2.21) we have

$$
\begin{aligned}
h^{2}(\mathbf{u}, \mathbf{u}) & \geqq \iint_{R} u^{2} d A-\frac{h^{2}}{\pi^{2}} \iint_{R}|\nabla u|^{2} d A \\
& =1-\frac{h^{2}}{\pi^{2}} \lambda-\left(\iint_{\bar{R}} u^{2} d A-\frac{h^{2}}{\pi^{2}} \iint_{\bar{R}}|\nabla u|^{2} d A\right) .
\end{aligned}
$$


We now show the term in parentheses is negative. For we observe $\bar{R}$ is the union of four kinds of disjoint regions (Fig. 6): (1) $h / 2 \times h$ rectangles, (2) $h / 4 \times h / 4$ squares, (3) $h \times h$ right triangles, (4) $h / 2$-size $\mathcal{L}$-shaped regions. On each of these regions, $R_{i}, u$ vanishes on an appropriate side, enabling one to reflect $u$ negatively in this side and to extend its definition to an $h \times h$ square on which the extension has mean value zero. The argument leading to (2.21) now applies and yields; for each $R_{i}$

$$
\iint_{R_{i}} u^{2} d A-\frac{h^{2}}{\pi^{2}} \iint_{R_{i}}|\nabla u|^{2} d A \leqq 0 .
$$

We conclude that the parenthetical term above is indeed negative, and hence

$$
h^{2}(\mathbf{u}, \mathbf{u}) \geqq 1-h^{2} \lambda / \pi^{2} .
$$

To prove (1.3) we examine the new numerator. $\sigma_{H}$ may be redefined as

$$
\bar{u}^{2}\left(\mathbf{x}_{1}\right)+\sum_{i=1}^{n-1}\left(\bar{u}\left(\mathbf{x}_{i+1}\right)-\bar{u}\left(\mathbf{x}_{i}\right)\right)^{2}+\bar{u}^{2}\left(\mathbf{x}_{n}\right)
$$

and $\sigma_{V}$ similarly. Appendix II shows that this time $N(\mathbf{u})=\sum \sigma_{I I}+\sum \sigma_{V}$.

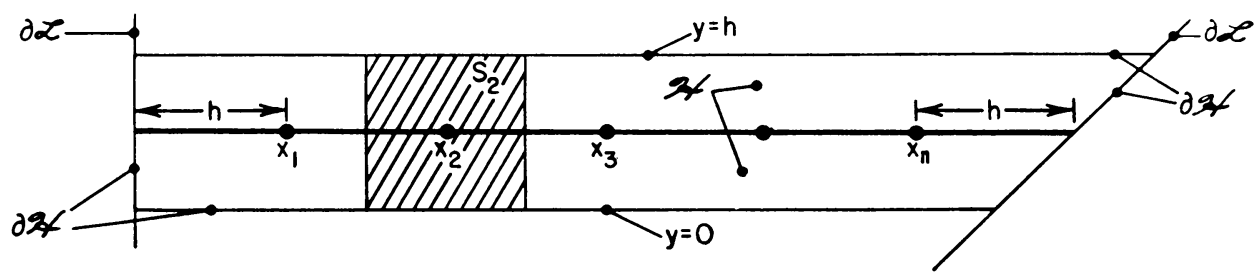

Figure 7

$\mathcal{F}$ is no longer the union of the $S\left(\mathbf{x}_{i}\right), \mathbf{x}_{i} \in H$. We define new $\phi_{i}$ (Fig. 8):

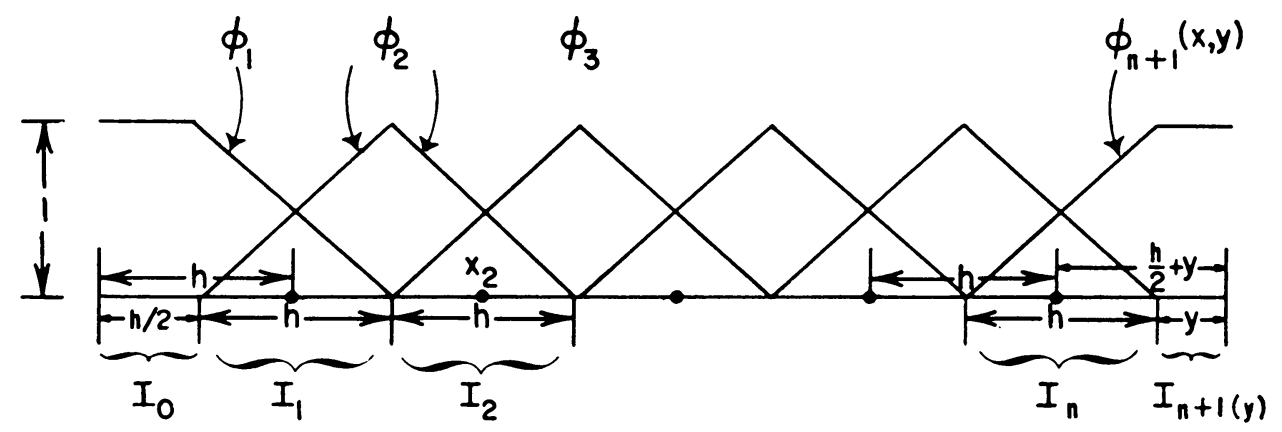

Figure 8

N.B.: length $\left(I_{n+1}\right)=y, y \in[0, h]$, and that $\phi_{n+1}$ is a function of $x$ and $y$. We have to account mainly for $\bar{u}^{2}\left(\mathbf{x}_{1}\right)$ and for $\bar{u}^{2}\left(\mathbf{x}_{n}\right)$.

$$
\int_{I_{1}} \frac{u}{h} d x=-\int_{I \cdot \cup I_{1}} u \frac{d \phi_{1}}{d x}=\int_{I_{n \cup I_{1}}} u_{x} \phi_{1} d x
$$

because $u \equiv 0$ on the left side of $\partial \mathcal{H}$. 
Hence

$$
\bar{u}^{2}\left(\mathbf{x}_{1}\right)=\int_{0}^{h} \int_{I_{1}} \frac{u d A}{h^{2}} \leqq \frac{1}{h^{2}} \int_{0}^{h} \int_{I_{0} \cup I_{1}} \phi_{1} d A \iint u_{x}^{2} \phi_{1} d A=\int_{0}^{h} \int_{I_{0} \cup I_{1}} u_{x}^{2} \phi_{1} d A
$$

Similarly, because $u \equiv 0$ on the right side of $\partial \mathfrak{F}$,

$$
\int_{I_{n}} \frac{u}{h} d x=-\int_{I_{n} \cup I_{n+1}(y)} u \frac{\partial \phi_{n+1}(x, y)}{\partial x} d x=\int_{I_{n} \cup I_{n+1}(y)} u_{x} \phi_{n+1}(x, y) d x
$$

and thus

$$
\begin{aligned}
\bar{u}^{2}\left(\mathbf{x}_{n}\right) & =\left(\int_{0}^{h} \int_{I_{n}} \frac{u}{h^{2}} d x d y\right)^{2} \\
& \leqq \frac{1}{h^{2}} \int_{0}^{h} \int_{I_{n} \cup I_{n+1}(y)} \phi_{n+1}(x, y) d x d y \int_{0}^{h} \int_{I_{n} \cup I_{n+1}(y)} \phi_{n+1}(x, y) u_{x}^{2} d x d y \\
& =\int_{0}^{h} \int_{I_{n} \cup I_{n+1}(y)} \phi_{n+1}(x, y) u_{x}^{2} d x d y .
\end{aligned}
$$

The interior part of $\sigma_{H}$ is handled as before; again the $\phi$ 's add to 1 on $\mathcal{H}$ and

$$
\sigma_{H} \leqq \iint_{\mathfrak{F C}} u_{x}^{2} d x d y
$$

It should be clear how to define the $\phi$ 's to verify, similarly, that

$$
\sigma_{V} \leqq \iint_{v} u_{y}^{2} d x d y \text {. }
$$

Summing, it follows again that

$$
N(\mathbf{u}) \leqq \lambda .
$$

Hence, with $\lambda$ the first eigenvalue of the $\mathscr{L}$,

$$
\lambda \leqq \lambda_{h} \leqq \lambda /\left(1-\operatorname{ch}^{2} \lambda\right), \quad c=1 / 4+1 / \pi^{2} .
$$

For the full $L$ one runs into trouble with the strip $\mathcal{F}$ in Fig. 9 , for $u \not \neq 0$ on the left side of $\partial \mathcal{H}$. We have been unable to modify the procedure leading to (3.3) to conclude even that $N(\mathbf{u}) \leqq \lambda+O\left(h^{2}\right)$.

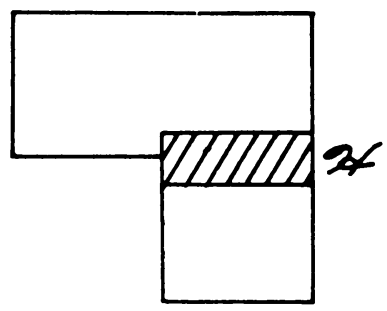

Figure 9

4. $\Delta u=-\lambda u$ in $\mathscr{L}, \partial u / \partial \nu=0$ on $\partial \mathfrak{L}$. We use a full $L$ as indicated in Figure 10, where the level lines of the piecewise linear functions to be used have also been 
sketched. All functions are assumed symmetric in the diagonal and orthogonal to the constant functions.

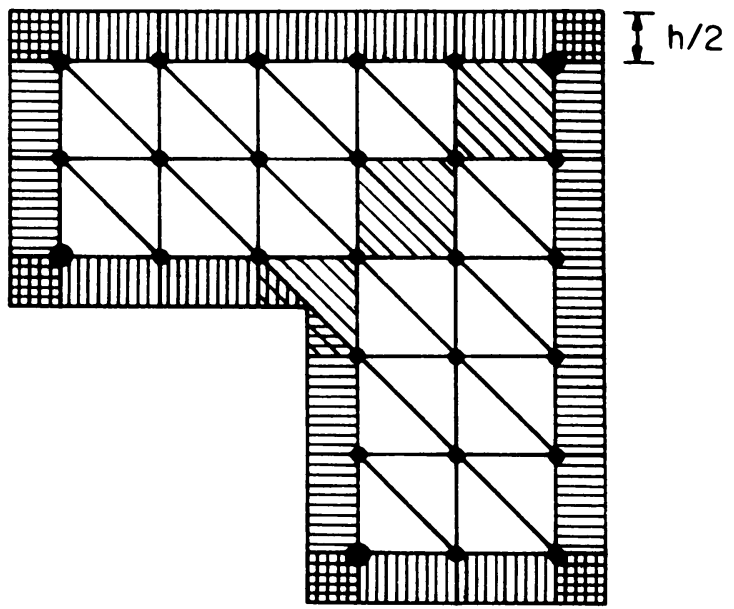

Figure 10

Appendix III shows two things: if $V_{M}=\max (|v|$ at the three enlarged dots in Fig. 10), then

$$
D(\mathbf{v}) \geqq h^{2}(\mathbf{v}, \mathbf{v})-h^{2}\left[N(\mathbf{v})+V_{M}^{2}\right] / 4 ;
$$

and that $N(\mathbf{v})$ consists only of a sum of squares of differences of neighboring values of $\mathbf{v}$. Thus, if $u$ is normalized over the full $L$,

$$
N(\mathbf{u}) \leqq \lambda
$$

by the method of Section 2 . (Since $N(\mathbf{u})$ contains only squared differences, no information about $u$ on $\partial L$ is required.) Also by Section 2 ,

$$
h^{2}(\mathbf{u}, \mathbf{u}) \geqq 1-h^{2} \lambda / \pi^{2} .
$$

Appendix IV shows that the mean values of $u$ (over the $h \times h$ squares centered on the three dots) are bounded by an explicit increasing function of $\lambda, f(\lambda, h)$ (Eq. (IV.2)). From this and (4.1)-(4.3) we conclude

$$
\lambda \leqq \lambda_{h} \leqq \lambda /\left[1-\operatorname{ch}^{2} \lambda-h^{2} f(\lambda, h) / 4\right], \quad c=1 / 4+1 / \pi^{2} .
$$

5. The Higher Eigenvalues. The min-max principle is, with $\sum$ running from 1 to $k$ on $i$,

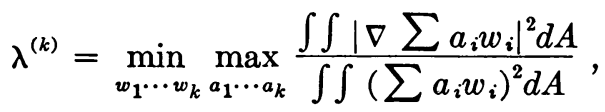

where $w_{1} \cdots w_{k}$ are piecewise differentiable, linearly independent functions over the domain which satisfy the boundary conditions. A larger min-max is obtained by restricting attention to all sets $\left\{v_{1}, \cdots, v_{k}\right\}$ of linearly independent piecewise linear functions satisfying the boundary conditions; and an upper bound $\lambda_{h}{ }^{(k)}$ results: 


$$
\lambda^{(k)} \leqq \min _{\mathbf{v}_{1} \cdots v_{k}} \max _{a_{1} \cdots a_{k}} \frac{\iint\left|\nabla \sum a_{i} v_{i}\right|^{2} d A}{\iint\left(\sum a_{i} v_{i}\right)^{2} d A}=\min _{\mathbf{v}_{1} \cdots \mathbf{v}_{k}} \max _{a_{1} \cdots a_{k}} \frac{N\left(\sum a_{i} \mathbf{v}_{i}\right)}{D\left(\sum a_{i} \mathbf{v}_{i}\right)} \equiv \lambda_{h}{ }^{(k)}
$$

(one notes for the last that $\nabla_{1} \cdots \nabla_{k}$ are linearly independent if and only if $v_{1} \cdots v_{k}$ are linearly independent). $\lambda_{h}{ }^{(k)}$ is also the $k$ th eigenvalue of the problem $A \mathbf{v}=\lambda B \mathbf{v}$; this can be seen by noting that $B^{-1} A$ is self-adjoint using $(\mathbf{v}, \mathbf{w})_{B} \equiv(\mathbf{v}, B \mathbf{w})$ and applying [20, p. 181]. Now set $\mathbf{u}_{i}=\left\{\iint_{S_{j}} u_{i} / h^{2}\right\}$ with $u_{i}$ the $i$ th eigenfunction normalized so that $\iint u_{i}{ }^{2} d A=1$ and orthogonalized so $\iint u_{m} u_{n} d A=0, m \neq n$.

Under the assumption that $\mathbf{u}_{1} \cdots \mathbf{u}_{k}$ are linearly independent we have

$$
\lambda_{h}{ }^{(k)} \leqq \max _{a_{1} \cdots a_{k} ; \Sigma a_{i}{ }^{2}=1} \frac{N\left(\sum a_{i} \mathbf{u}_{i}\right)}{D\left(\sum a_{i} \mathbf{u}_{i}\right)} .
$$

By the previous work in Sections 2 and 3 , for each $a_{1}, \cdots, a_{k}$

$$
\frac{N\left(\sum a_{i} \mathbf{u}_{i}\right)}{D\left(\sum a_{i} \mathbf{u}_{i}\right)} \leqq \frac{\iint\left|\nabla \sum a_{i} u_{i}\right|^{2} d A}{\iint\left(\sum a_{i} u_{i}\right)^{2} d A-\operatorname{ch}^{2} \iint\left|\nabla \sum a_{i} u_{i}\right|^{2} d A}=\frac{\sum \lambda^{(i)} a_{i}{ }^{2}}{1-c h^{2} \sum \lambda^{(i)} a_{i}{ }^{2}} .
$$

Hence

$$
\lambda_{h}{ }^{(k)} \leqq \max _{a_{1} \cdots a_{k} ; \Sigma a_{i}{ }^{2}=1} \frac{\sum \lambda^{(i)} a_{i}{ }^{2}}{1-c h^{2} \sum \lambda^{(i)} a_{i}{ }^{2}} .
$$

But $0 \leqq f \leqq f_{\max }$ and $k>0$ implies $\max [f /(1-k f)]=f_{\max } /\left(1-k f_{\max }\right)$. Hence

$$
\lambda^{(k)} \leqq \lambda_{h}^{(k)} \leqq \lambda^{(k)} /\left(1-\operatorname{ch}^{2} \lambda^{(k)}\right)
$$

and (1.6) follows.

Finally, we show that for fixed $k, \mathbf{u}_{1} \cdots \mathbf{u}_{k}$ are linearly independent as $h \rightarrow 0$. For suppose $\sum a_{i} \mathbf{u}_{i}=0, \sum a_{i}{ }^{2}=1$. From (1.1) and the procedures leading to (1.2)

$$
\begin{aligned}
D\left(\sum a_{i} \mathbf{u}_{i}\right) & \geqq \iint\left(\sum a_{i} u_{i}\right)^{2} d A-c h^{2} \iint\left|\nabla \sum a_{i} u_{i}\right|^{2} d A \\
& \geqq 1-\operatorname{ch}^{2} \sum \lambda^{(i)} a_{i}{ }^{2}, \quad c=1 / 4+1 / \pi^{2} .
\end{aligned}
$$

Let $c h^{2}<1 / \lambda^{(k)}$. Then $D\left(\sum a_{i} \mathbf{u}_{i}\right)>0$. But $D$ is positive definite, hence $\sum a_{i} \mathbf{u}_{i}$ $\neq 0$, a contradiction of the linear dependence of the $\mathbf{u}_{i}, i=1, \cdots, k$.

It is easily verified that the additional term, introduced in the denominators when considering the "Neumann" eigenfunctions of Section 4, causes little difficulty, and results in

$$
\lambda^{(k)} \leqq \lambda_{h}{ }^{(k)} \leqq \lambda^{(k)} /\left[1-c h^{2}-h^{2} \sum_{i=1}^{k} f\left(\lambda^{(i)}, h\right) / 4\right] \equiv g_{h}{ }^{(k)}\left(\lambda^{(1)}, \cdots, \lambda^{(k)}\right)
$$

6. Upper Bound Using Standard Matrix Eigenvalues. The usual approach to the membrane's eigenvalues is to solve the matrix eigenvalue problem

$$
A \mathrm{v}^{*}=\lambda_{h}{ }^{*} h^{2} \mathrm{v}^{*}
$$

instead of the problem indicated in Section 1 as $A \mathbf{v}=\lambda_{h} B \mathbf{v}$.

Since $v^{*}$ may be substituted in the Rayleigh quotient, we have by (1.1), that

$$
\begin{aligned}
\lambda \leqq N\left(\mathbf{v}^{*}\right) / D\left(\mathbf{v}^{*}\right) & \leqq N\left(\mathbf{v}^{*}\right) /\left[h^{2}\left(\mathbf{v}^{*}, \mathbf{v}^{*}\right)-h^{2} N\left(\mathbf{v}^{*}\right) / 4\right] \\
& =\lambda_{h}{ }^{*} /\left(1-h^{2} \lambda_{h}{ }^{*} / 4\right)
\end{aligned}
$$


giving a strict upper bound on the lowest eigenvalue. For the mesh of Section 3, [4, p. 68] shows

$$
\lambda_{h}^{*}=\lambda-\gamma h^{2}+o\left(h^{2}\right), \quad h \rightarrow 0 ;
$$

thus $\lambda_{h}{ }^{*}$ is an asymptotic lower bound, not a strict lower bound.

7. A Numerical Example. The lowest eigenvalues of both the $\mathscr{L}$ and the $L$, corresponding to $u=0$ on the boundary, were approximated by the Rayleigh-Ritz technique using the mesh of Section 3. A suitable generalization of the successive overrelaxation technique used by Moler [4, pp. 114-117] was programmed to solve $A \mathbf{v}=$ $\lambda_{h} B v$. The Rayleigh quotients were computed in double precision to obtain sufficient accuracy in the matrix eigenvalues. Values of $1 / h$ were $8(4) 36$ and 42 (Table 1 ).

\section{TABle 1}

Rayleigh-Ritz eigenvalues, standard mesh, $u=0$ on boundary

\begin{tabular}{|c|c|c|c|c|}
\hline \multicolumn{4}{|c|}{ Half- $L, \mathfrak{L}$} & \multirow{2}{*}{$\frac{L}{\underset{\text { Upper bound }}{\lambda_{h}}}$} \\
\hline $1 / h$ & $\begin{array}{c}\text { Upper bound } \\
\lambda_{h}\end{array}$ & $\begin{array}{l}\text { Lower bound } \\
\lambda_{h} /\left(1+\operatorname{ch}^{2} \lambda_{h}\right)\end{array}$ & Difference & \\
\hline 8 & 15.5572882 & 14.334 & 1.224 & 9.9659766 \\
\hline 12 & 15.3580389 & 14.804 & 0.555 & 9.8028565 \\
\hline 16 & 15.2879549 & 14.974 & 0.314 & 9.4081708 \\
\hline 20 & 15.2554144 & 15.054 & 0.202 & 9.7099600 \\
\hline 24 & 15.2376994 & 15.098 & 0.140 & 9.6921083 \\
\hline 28 & 15.2270005 & 15.124 & 0.103 & 9.6807234 \\
\hline 32 & 15.2200476 & 15.141 & 0.079 & 9.6729507 \\
\hline 36 & 15.2152759 & 15.153 & 0.062 & 9.6673700 \\
\hline 42 & 15.2105050 & 15.165 & 0.046 & 9.6615029 \\
\hline
\end{tabular}

TABLE 2

Previously computed standard eigenvalues, source indicated

\begin{tabular}{r|c|r|r|r}
\hline \multicolumn{2}{c|}{ Half- $L, \&$ (Bowdler \& Wilkinson) } & \multicolumn{2}{|c}{$L$ (mostly Moler) } \\
\cline { 2 - 4 } $1 / h$ & $\lambda_{h}{ }^{*}$ & $\begin{array}{c}\text { Upper bound } \\
\lambda_{h}{ }^{*} /\left(1-h^{2} \lambda_{h}{ }^{*} / 4\right)\end{array}$ & $1 / h$ & $\lambda_{h}{ }^{*}$ \\
\hline 6 & 14.8325923 & 16.536 & 4 & $9.64142546(\mathrm{~B} \& W)$ \\
8 & 14.9931528 & 15.926 & 8 & $9.69316221(\mathrm{~B} \& W)$ \\
10 & 15.0671631 & 15.657 & 10 & 9.68829145 \\
11 & 15.0899260 & 15.576 & 20 & 9.66696983 \\
12 & 15.1072074 & 15.515 & 30 & 9.65743368 \\
13 & 15.1206339 & 15.467 & 40 & 9.65249358 \\
14 & 15.1312711 & 15.430 & 50 & 9.64954711 \\
15 & 15.1398404 & 15.399 & 80 & 9.64527693 \\
16 & 15.1468447 & 15.375 & 100 & 9.64393241 \\
\hline
\end{tabular}


The lowest eigenvalue of (6.1) has been computed for the $L$ by Moler [4, p. 124] with $1 / h=10(10) 100$. Bowdler and Wilkinson at the National Physical Laboratory calculated the lowest eigenvalue of (6.1) for the $\mathcal{L}$ and the $L$ (unpublished, 1962) with $1 / h=6(1) 16$. See also [12, p. $841 \mathrm{ff}]$, where two methods are discussed which significantly improve on the order of convergence to the first eigenvalue of the $L$ (Table 2).

To more easily compare these results, all four sets of eigenvalues were fit, as functions of $h$, by a nonlinear least-squares routine which could handle approximations of the form $\sum a_{i} h^{b_{i}}, a_{i}$ and $b_{i}$ parameters. The best fits were

$\begin{array}{lcl}\text { Source } & \text { Region } & \text { Fit to } \lambda_{h}=\lambda(h) \\ \text { Moler } & L & \lambda_{L}+2.2 h^{4 / 3}-5.2 h^{2}-h^{3.1} \\ \text { Author } & L & \lambda_{L}+2.2 h^{4 / 3}+11.8 h^{2}+11 \mathrm{~h}^{3.6} \\ \text { NPL } & \mathscr{L} & \lambda_{\mathscr{L}}-13 h^{2}+h^{3} \\ \text { Author } & \mathscr{L} & \lambda_{\mathcal{L}}+23 h^{2}-2 h^{3}\end{array}$

Assuming $\lambda_{\mathcal{L}}$ known (below), (3.4) predicts a priori an upper bound behaving like $\lambda_{£}+81 h^{2}$. $\lambda_{\&}$ was 15.197 for the NPL eigenvalues and 15.1973 for the author's, while $\lambda_{L}$ was 9.639724 for both Moler's and the author's eigenvalues. From the results for the $\mathfrak{L}$ it is easily seen that the $a$ posteriori lower bound derived from (3.4) behaves like $\lambda-58 h^{2}$ while the $a$ posteriori upper bound (6.2) behaves like $\lambda+44 h^{2}$.

Thanks are due to Connie Luders for preparing the diagrams and to the referee for his help, especially with Appendix IV.

Appendix I. Numerator as a sum of squares of differences:

Notation: $+-2-+$ means $2[v \text { (right) }-v \text { (left) }]^{2}$ occurs in the numerator.

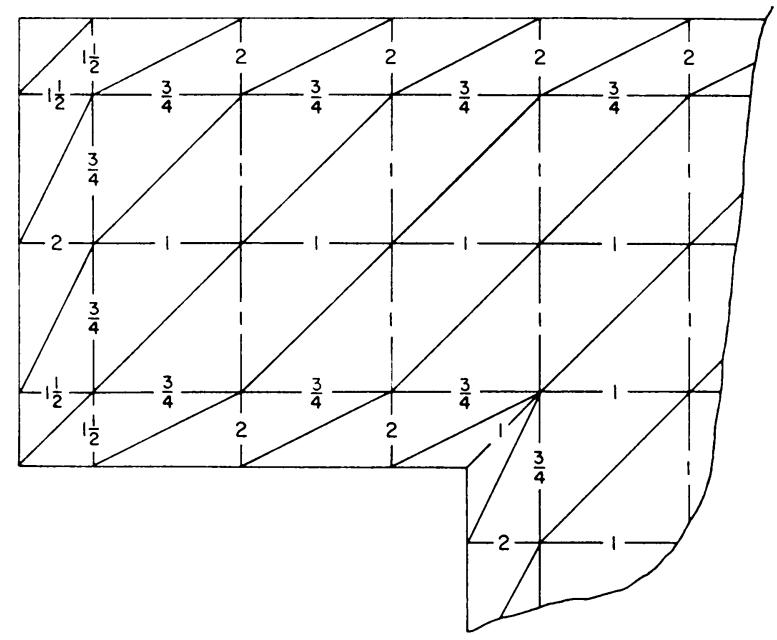

This is easily verified by squaring the (constant) gradient above each triangle, multiplying by the area of the triangle, and adding up over the triangles.

There is no loss in generality in considering the case of the large $h$ shown here and in what follows, for the forms do not change with decreasing $h$.

All diagrams which follow (in the next two appendices as well as this one) consider various quadratic forms using the notation to be described next. 
Numerator as a quadratic form:

Notation: $-4-$ means $4 v^{2}$ (vertex) occurs in the form;

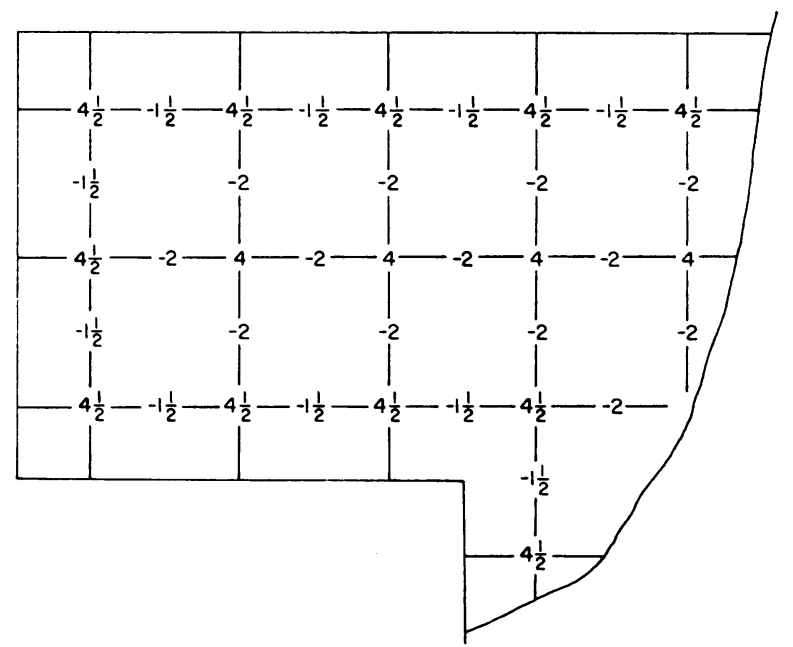

The denominator. If a linear function, $P(\mathbf{x})$, defined on a triangle, $T$, of base $h$ and altitude $k$, has values $a, b$, and $c$ above each vertex then

$$
\iint_{T} P^{2} d A=\frac{h k}{12}\left(a^{2}+b^{2}+c^{2}+a b+a c+b c\right) .
$$

For example: to find the coefficient of $v^{2}$ ("SW corner") we examine the contribution of the six triangles containing that corner (see insert). Hence the denominator is $h^{2}$ (following form)/12:

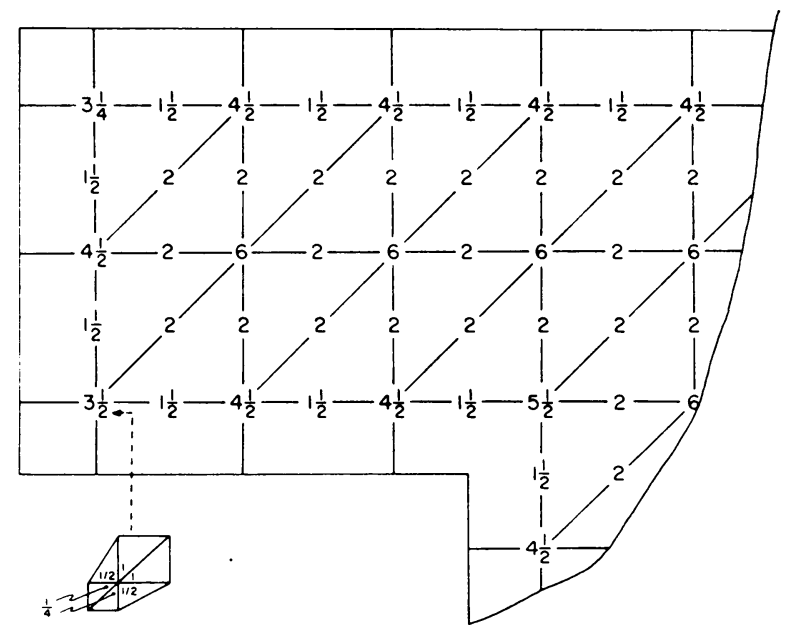

Thus $h^{2}(\mathbf{v}, \mathbf{v})=D(\mathbf{v})+h^{2}$ (following form) $/ 12$ : 


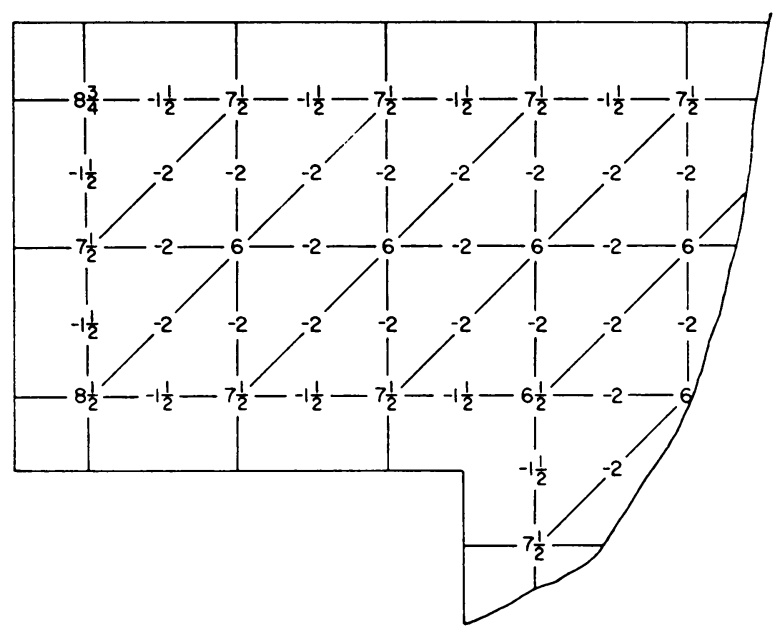

This last form is the sum of $A+B$, where $A=\sum$ (diagonal differences) $^{2}$.
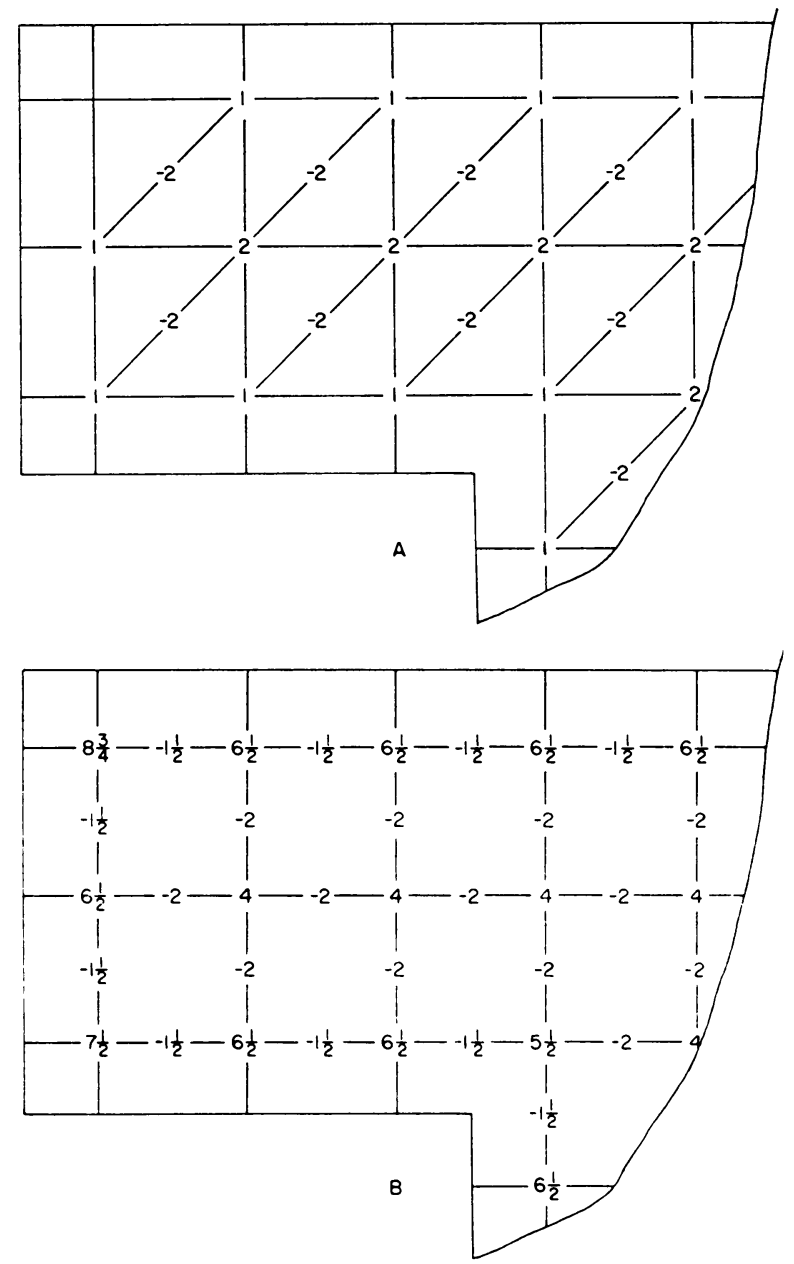
From the inequality $(a-c)^{2} \leqq 2\left[(a-b)^{2}+(b-c)^{2}\right]$, or $(a-c)^{2} \leqq(a-d)^{2}+$ $(d-b)^{2}+(a-b)^{2}+(b-c)^{2}, A \leqq$ following form:

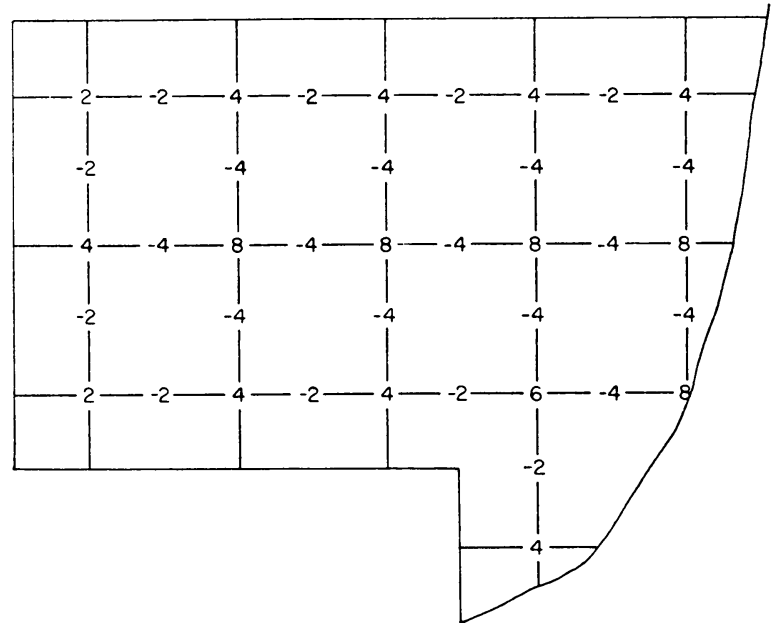

Hence $h^{2}(\mathbf{v}, \mathbf{v}) \leqq D(\mathbf{v})+h^{2}$ (following form) $/ 12$ :

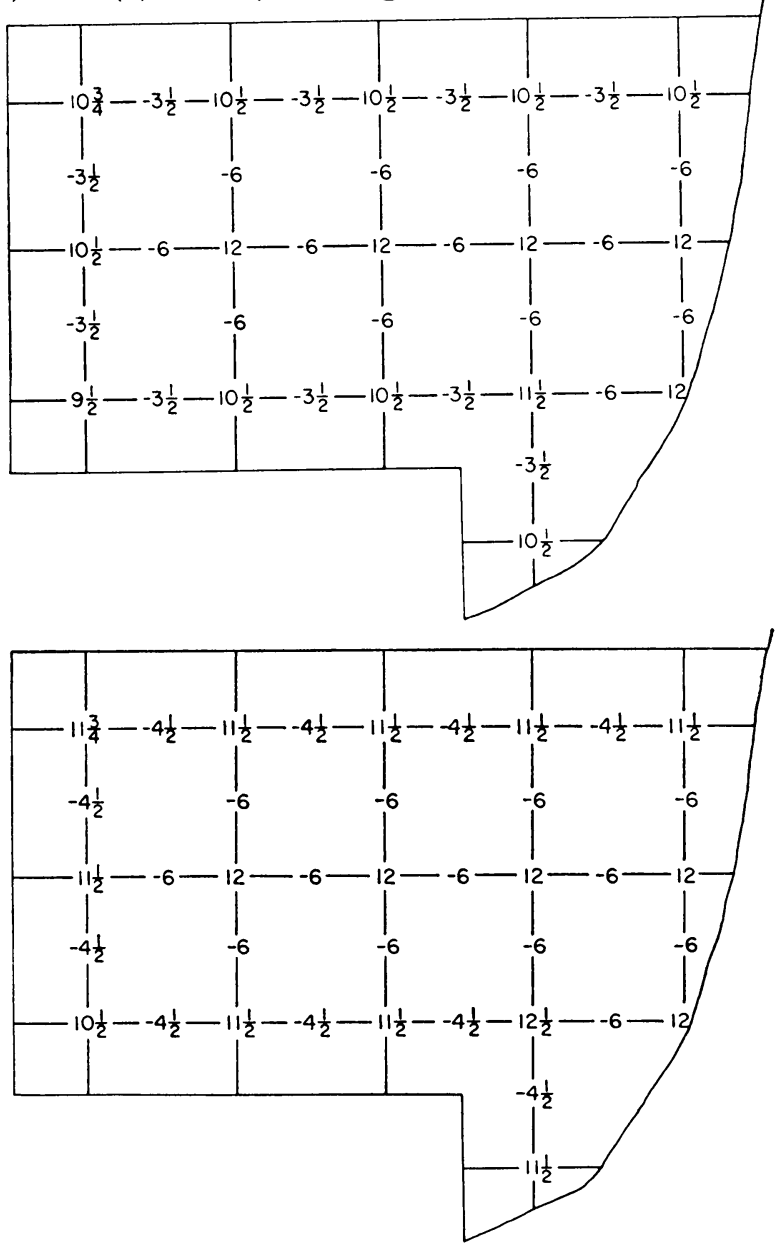


Now, adding $\frac{1}{2} \sum_{\text {bdry }}$ ("boundary differences") ${ }^{2}$, where a "boundary difference" is the difference of values at two neighboring points, each adjacent to the boundary, $h^{2}(\mathbf{v}, \mathbf{v}) \leqq D(\mathbf{v})+h^{2}$ (preceding form) $/ 12$.

Finally, by adding appropriate squares of "boundary values," we have

$$
\begin{aligned}
h^{2}(\mathbf{v}, \mathbf{v}) & \leqq D(\mathbf{v})+h^{2}(3 \cdot \text { Numerator }) / 12, \quad \text { or } \\
D(\mathbf{v}) & \geqq h^{2}(\mathbf{v}, \mathbf{v})-h^{2} N(\mathbf{v}) / 4 .
\end{aligned}
$$

APPENDIX II. Numerator as a quadratic form. With the notation of Appendix I, $N(\mathbf{v})$ is the following form:

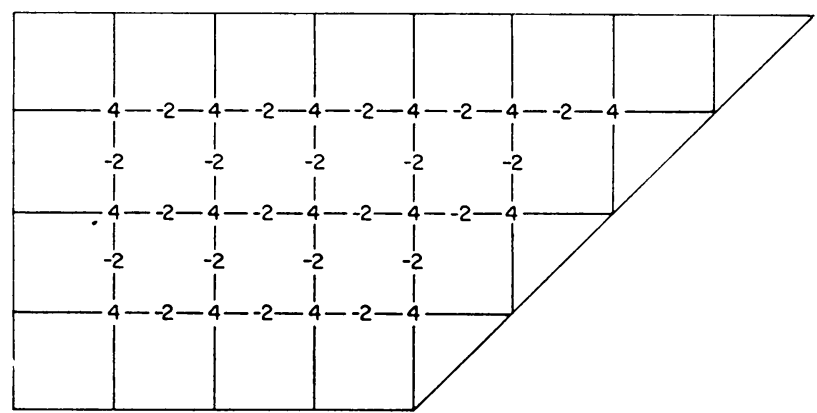

Denominator as a quadratic form. $D(\mathbf{v})=h^{2}$ (following form) $/ 12$ :

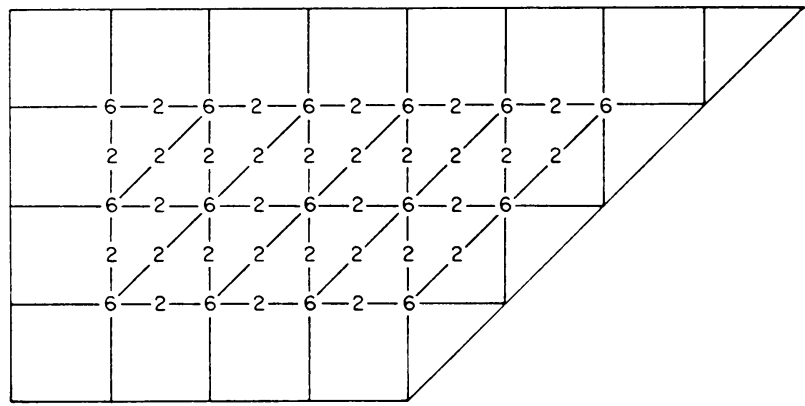

Performing manipulations as in Appendix I we arrive at the conclusion that $h^{2}(\mathbf{v}, \mathbf{v}) \leqq D(\mathbf{v})+h^{2}$ (following form) $/ 12$ :

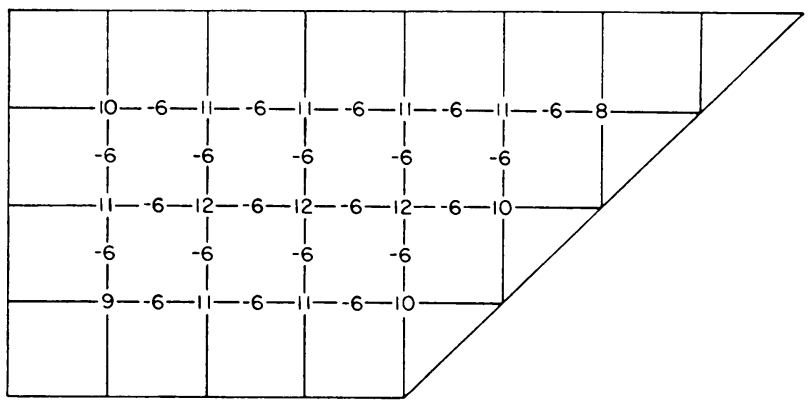


Hence

$$
h^{2}(\mathbf{v}, \mathbf{v}) \leqq D(\mathbf{v})+h^{2}(3 N(\mathbf{v})) / 12
$$

or

$$
D(\mathbf{v}) \geqq h^{2}(\mathbf{v}, \mathbf{v})-h^{2} N(\mathbf{v}) / 4 .
$$

APPENDIX III. In deriving and manipulating the quadratic forms to follow, careful advantage is taken of the level lines of $v(x)$ (Fig. 10) and the symmetry of $v(x)$ in the diagonal of $L$.

$N(\mathbf{v})$ is the following form:

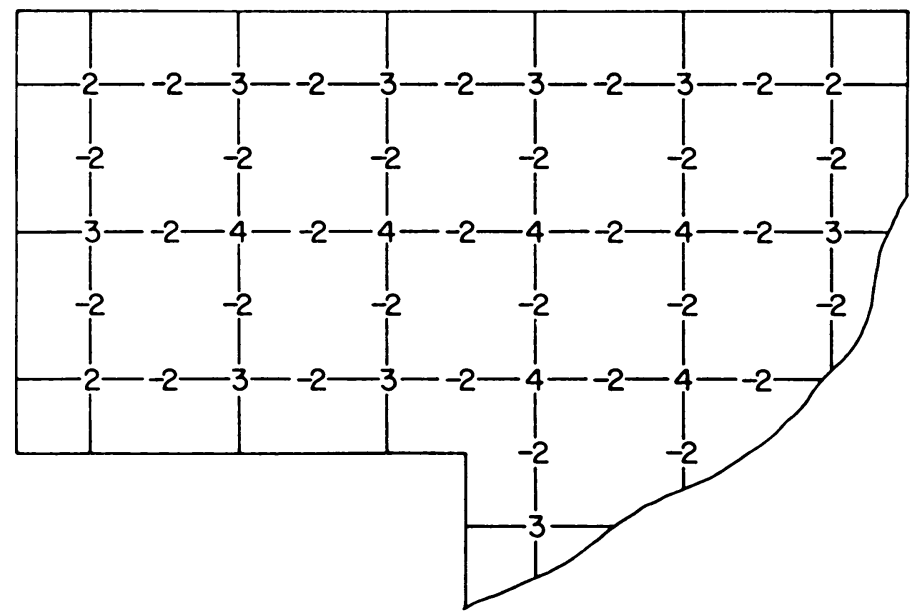

$D(\mathbf{v})$ is the following form:

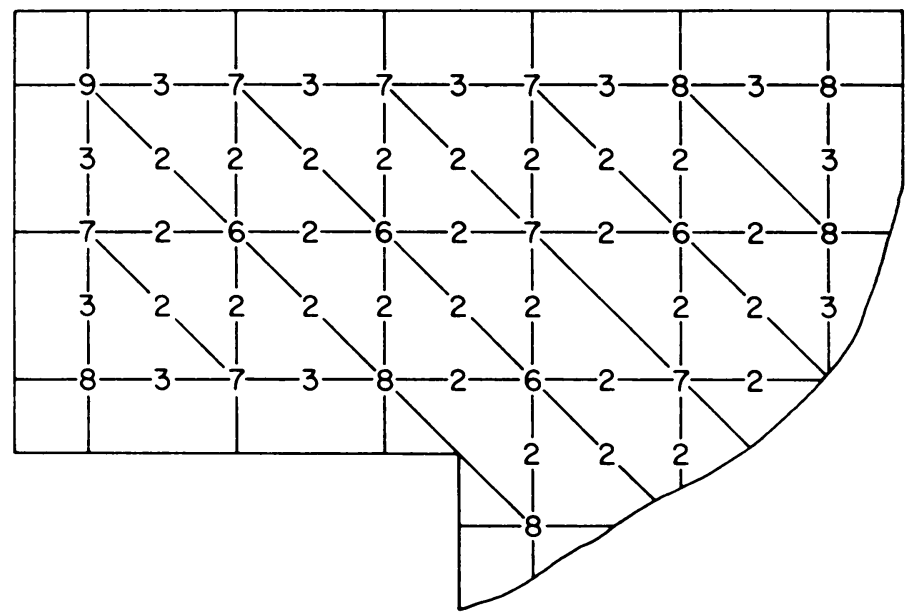

Using some care in manipulating $D$ as in Appendix I, one finds $h^{2}(\mathbf{v}, \mathbf{v}) \leqq D(\mathbf{v})+$ 
$h^{2}$ (following form)/12:

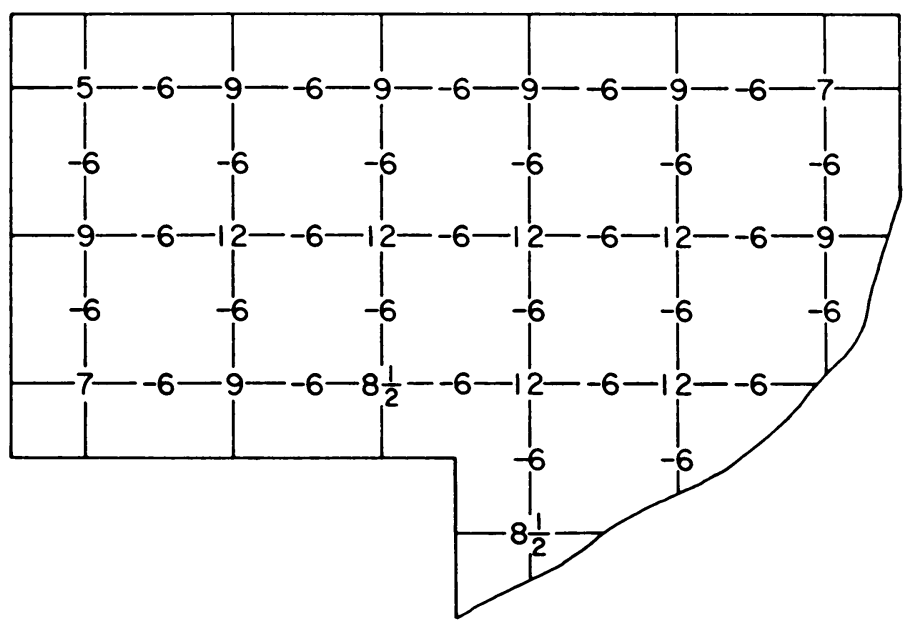

Thus $h^{2}(\mathbf{v}, \mathbf{v}) \leqq D(\mathbf{v})+h^{2} N(\mathbf{v}) / 4+h^{2} V / 12$, where $V$ is the sum of the squares of $\mathbf{v}$ at the three heavy dots in Fig. 10. Equation (4.1) follows.

Appendix IV. Bounds on Eigenfunctions. It is easy to find explicit $O(\lambda)$ bounds $(\lambda \rightarrow \infty)$ on $\max _{D}|u|$ if $u$ satisfies $\Delta u=-\lambda u$ in $D, u=0$ on $\partial D, \iint_{D} u^{2}=1$. For $u$ satisfies an integral equation involving the Green's function of $D$, from which the Schwarz inequality yields $\max |u|^{2} \leqq \lambda^{2} \max \iint G^{2}$. Because $G$ is an increasing function of $D$, an explicit bound can be found by surrounding $D$ with a rectangle and expanding the rectangle's Green's function in terms of its eigenfunctions. This $O(\lambda)$ bound can be improved to $\max |u| \leqq(4 \lambda / \pi)^{1 / 2}$ by using the Green's function for $\Delta u$ $-p u, p>0$ and optimizing the resulting bound by choosing $p=\lambda$.

That such a bound for a general domain must be $O\left(\lambda^{q}\right)(\lambda \rightarrow \infty)$ for some $q \geqq 1 / 4$ may be seen by considering the subsequence of normalized eigenfunctions for the unit circle: $J_{0}\left(\sqrt{ } \lambda_{k} r\right) /\left[\sqrt{ } \pi J_{0}{ }^{\prime}\left(\sqrt{ } \lambda_{k}\right)\right], \sqrt{ } \lambda_{k}=k$ th zero of $J_{0},[15$, p. 306], [18, p. 364].

When the boundary condition is $\partial u / \partial \nu=0$ (instead of $u=0$ ) the above method fails, for the behaviour of the Neumann's function (or even the kernel function) is not easily tied to the relation of the domains involved in their definition. Another procedure is called for, then, and is based on a mean-value theorem.

The mean-value theorem, for regular function, $u$, satisfying $\Delta u=-\lambda u$ inside a circle of radius $r$, is [16, p. 289, correcting a misprint]:

$$
\int_{0}^{2 \pi} u(r, \theta) r d \theta=2 \pi u(0,0) r J_{0}(\sqrt{ } \lambda r) .
$$

Multiplying by $J_{0}(\sqrt{ } \lambda r)$, integrating from 0 to $R$, and applying the Schwarz inequality, we have [15, p. 484, 11. 3.34]

$$
\begin{aligned}
u^{2}(0,0) & \leqq \int_{0}^{R} \int_{0}^{2 \pi} u^{2} r d \theta d r /\left[2 \pi \int_{0}^{R} J_{0}{ }^{2}(\sqrt{ } \lambda r) r d r\right] \\
& =\int_{0}^{R} \int_{0}^{2 \pi} u^{2} r d \theta d r /\left\{\pi R^{2}\left[J_{0}{ }^{2}(\sqrt{ } \lambda R)+J_{1}^{2}(\sqrt{ } \lambda R)\right]\right\} .
\end{aligned}
$$

(This bound is sharp for the eigenfunctions of the unit circle mentioned above.) It is 
of no use, however, to let $R \rightarrow 0$ to obtain a uniform bound on $|u|$; using the analogue (for circles) of (2.21) to bound the integral in (IV.1) (in terms of $u^{2}(0,0)$ and $\lambda$ ) only yields $u^{2}(0,0) \leqq u^{2}(0,0)+$ something.

To obtain a usable bound, then, of $|u|$ at the three large dots in Fig. 10, let us first focus attention on the dot adjacent to the $90^{\circ}$ corner. Reflection of the $\mathfrak{L}$ (and $u$ ) three times around the corner defines a new region, $\mathfrak{L}_{4}$, and a new function, $\tilde{u}$. Assuming $\Delta \tilde{u}=-\lambda \tilde{u}$ in $\mathfrak{L}_{4}$ (this assumption is discussed below) one gains the analyticity of $\tilde{u}$ in $\mathfrak{L}_{4}$ and assures the validity of (IV.1) for any disc contained in $\mathfrak{L}_{4}$. Placing $(0,0)$ at the dot, and recalling $u$ is normalized over the full $L$, (IV.1) yields $u^{2}(d o t) \leqq 2 /\left\{\pi R^{2}\left[J_{0}{ }^{2}(\sqrt{ } \lambda R)+J_{1}{ }^{2}(\sqrt{ } \lambda R)\right]\right\}$ for any $R<1-h$. Under similar assumptions, $u$ at the other two dots may be bounded by reflecting three or seven times, the conclusion being

$$
\begin{aligned}
\left.\max \left[u^{2} \text { (three dots }\right)\right] & \leqq 4 /\left\{\pi R^{2}\left[J_{0}{ }^{2}(\sqrt{ } \lambda R)+J_{1}{ }^{2}(\sqrt{ } \lambda R)\right]\right\} \\
& \equiv f(\lambda, h), \quad R=1-h .
\end{aligned}
$$

We see $f(0, h)=4 /\left[\pi(1-h)^{2}\right]$; and that for fixed $h, f=O(\sqrt{ } \lambda), \lambda \rightarrow \infty$. Furthermore, since $\partial f / \partial \sqrt{ } \lambda=2 \pi R^{2} J_{1}{ }^{2}(\sqrt{ } \lambda R) /\left(\sqrt{ } \lambda f^{2}\right) \geqq 0$ (using $J_{0}{ }^{\prime}(z)=-J_{1}(z), J_{1}{ }^{\prime}(z)=$ $J_{0}(z)-J_{1}(z) / z$ ), we see $f$ is increasing in $\lambda$ (for fixed $h$ ) and so may be used in obtaining the lower bound (1.5a).

Finally, (IV.2) is seen to bound $u$ uniformly in the $h \times h$ squares centered on the three dots, and thus bounds its average over these squares.

To show that $\Delta \tilde{u}=-\lambda \tilde{u}$ in $\mathfrak{L}_{4}$, choose a disc $D$, centered on the $90^{\circ}$ corner of $\mathfrak{L}$, of radius small enough so that the lowest eigenvalue $\mu_{1}$ of the problem $\Delta v=-\mu_{1} v$ in $D, v=0$ on $\partial D$ satisfies $\mu_{1}>\lambda$. Then the solution $u^{*}$ to $\Delta u^{*}=-\lambda u^{*}$ in $D, u^{*}=\tilde{u}$ on $\partial D$, is unique and satisfies $\partial u^{*} / \partial \nu=0$ on $\partial \mathscr{L} \cap D$. Since $\mu_{2}$, the lowest eigenvalue of the problem $\Delta v=-\mu_{2} v$ in $\mathscr{L} \cap D, v=0$ on $\partial D \cap \mathscr{L}, \partial v / \partial \nu=0$ on $\partial \mathscr{L} \cap D$ satisfies $\mu_{2}>\mu_{1}>\lambda ; u^{*} \equiv \tilde{u}$ in $\mathscr{L} \cap D$, and thus in $\mathscr{L}_{4} \cap D$. Similar arguments show $\tilde{u}$ is regular at every interior point of $\mathfrak{L}_{4}$; and that the corresponding reflection around the $45^{\circ}$ corner is also regular in its domain of eight $\mathscr{L}^{\prime} \mathrm{s}$.

Finally, it is worth pointing out that, had the hypotenuses of the triangles in Fig. 10 sloped like those in Fig. 2, it would have been necessary to bound $|u|$ in a neighborhood of the $135^{\circ}$ corner; and the reflections would have had to have been completed on a Riemann surface (where no mean value theorem is known to the author).

For discussions of pointwise bounds in general see, e.g., [17, p. 101] and its references.

University of California

Los Alamos Scientific Laboratory

Los Alamos, New Mexico

1. R. Courant, "Variational methods for the solutions of problems of equilibrium and vibrations," Bull. Amer. Math. Soc., v. 49, 1943, pp. 1-23. MR 4, 200.

2. L. Collatz, "Konvergenz des Differenzverfahrens bei Eigenwertproblemen partieller Differentialgleichungen," Deutsche Math., v. 3, 1938, pp. 200-212.

3. H. F. WEINBERGER, "Lower bounds for higher eigenvalues by finite difference methods," Pacific J. Math., v. 8, 1958, pp. 339-368. MR 21 \#6097.

4. C. Moler, Finite Difference Methods for the Eigenvalues of Laplace's Operator, Stanford Computer Science Report CS22, 1965. (Available from Defense Document Center, Cameron Station, Alexandria, Va.) 
5. J. Synge, The Hypercircle in Mathematical Physics: A Method for the Approximate Solution of Boundary Value Problems, Cambridge Univ. Press, New York, 1957, Sections 3.5-3.8 ff. MR 20 \#4073.

6. G. Pólya, "Sur une interprétation de la méthode des différences finies qui peut fournir des bornes supérieures ou inférieures," C. R. Acad. Sci. Paris, v. 235, 1952, pp. 995-997. MR 14, 656.

7. G. Pólya, "Estimates of eigenvalues," Studies in Mathematics and Mechanics Presented to Richard von Mises, Academic Press, New York, 1954, pp. 200-207. MR 16, 482.

8. G. Birkhoff, C. DE Boor, B. Swartz, \& B. Wendroff, "Rayleigh-Ritz approximation by piecewise cubic polynomials," SIAM J. Numer. Anal., v. 3, 1966, pp. 188-203. MR 34 \#3773.

9. G. Forsythe \& W. R. WAsow, Finite-Difference Methods for Partial Differential Equations, Wiley, New York, 1960, pp. 340-351. MR 23 \#B3156.

10. B. HUBBARD, "Bounds for eigenvalues of the free and fixed membrane by finite difference methods," Pacific J. Math., v. 11, 1961, pp. 559-590. MR 25 \#4633.

11. L. VEIDINGER, "Computation of the eigenvalues of a membrane by a finite-difference method," Z. Vycisl. Mat. i Mat. Fiz., v. 4, 1964, pp. 1037-1044=U.S.S.R. Comput. Math. and Math. Phys., v. 4, 1964. MR $30 \# 1620$.

12. J. K. REID \& J. E. WALSH, "An elliptic eigenvalue problem for a reentrant region," $J$. Soc. Indust. Appl. Math., v. 13, 1965, pp. 837-850. MR 31 \#6392.

13. J. Hersch, "Equations différentielles et fonctions de cellules," C. R. Acad. Sci. Paris, v. 240,1955 , pp. 1602-1604. MR 16, 929.

14. J. Hersch, "Lower bounds for all eigenvalues by cell functions: a refined form of H. F. Weinberger's method," Arch. Rational Mech. Anal., v. 12, 1963, pp. 361-366. MR $29 \# 745$.

15. R. Courant \& D. Hilbert, Methods of Mathematical Physics, Vol. I, Interscience, New York, 1953. MR 16, 426.

16. R. Courant \& D. Hilbert, Methods of Mathematical Physics, Vol. II, Interscience, New York, 1962. MR 25 \#4216.

17. L. E. Payne \& H. F. Weinberger, "Bounds for solutions of second order elliptic equations in terms of arbitrary vector fields," Arch. Rational Mech. Anal., v. 20, 1965, pp. 95-106. MR 33 \#401.

18. M. Abramowitz \& I. A. Stegun, (Editors), Handbook of Mathematical Functions with Formulas, Graphs and Mathematical Tables, National Bureau of Standards, Applied Math. Series, No. 55, U. S. Government Printing Office, Washington, D. C., reprint, 1965. MR 31 \#1400.

19. H. WEINBERGER, "Upper and lower bounds for eigenvalues by finite difference methods," Comm. Pure Appl. Math., v. 9, 1956, pp. 613-623. MR 18, 826.

20. P. Halmos, Finite-Dimensional Vector Spaces, Van Nostrand, Princeton, N. J., 1958. MR 19, 725 . 